\title{
Baseline-free damage identification of metallic sandwich panels with truss core based on vibration characteristics
}

Structural Health Monitoring

\author{
Lingling Lu, Hongwei Song, Wu Yuan and Chenguang Huang
}

\begin{abstract}
A baseline-free damage identification method is proposed to identify damages in metallic sandwich panels with truss core in the article. The method is based on flexibility matrix and gapped smoothing method, with damage index defined $D I_{m}$. The weight coefficient $m$ is introduced to consider the effect of damages on both low-order modes and highorder modes. Numerical simulations and experiments are conducted to evaluate the present method. Besides, damage index $D I_{m}^{*}$ is also defined by processing $D I_{m}$ with Teager energy operator, and comparisons between $D I_{m}$ and $D I_{m}^{*}$ are also carried out. Results show that the proposed method is effective in detecting single damage and multiple damages of the same or different extent. The weight coefficient $m$ plays a very important role in identification of multiple damages of different styles. When comparing with $D I_{m}^{*}$, it is found that the present index $D I_{m}$ is better at suppressing the singularity caused by contact nodes and detecting of multiple damages which contain small or slight damages.
\end{abstract}

\section{Keywords}

Sandwich panel with truss core, damage identification, gapped smoothing method, Teager energy operator, cell missing

\section{Introduction}

Structural health monitoring (SHM) deals with damage identification for aerospace, civil, and mechanical infrastructures, and SHM has gained considerable attentions toward diverse engineering applications., ${ }^{1,2}$ Sandwich structures consisting of two thin face sheets and a lightweight core $^{3-5}$ have been increasingly applied in industrial sectors such as ships, aircrafts, civil engineering, as well as aerospace engineering. As a class of newly developed lightweight multifunctional structures, sandwich panels with truss cores (SPTCs) have many superior properties, such as high specific bending stiffness, light weight, good thermal insulation, and acoustical isolation. ${ }^{6-9}$ Therefore, SPTCs are very promising candidates to be applied in the thermal protection systems (TPS) of high-speed aircrafts. When being used in TPS, damages may occur in the panel due to complicated aerodynamic force and harsh heat environment, and the damage may include buckling of the panel, ${ }^{10,11}$ breakage of truss, burn-through of face sheets, and so on. Besides, defects may also arise during the manufacturing process, for example, unbounded truss nodes to the face sheet. These damages and defects would alter the mechanical properties of the
SPTC, such as reducing the stiffness and strength, decreasing the threshold of buckling temperature, changing the natural frequencies and vibration modes, which may increase the risk of structural failure. Therefore, early detection and recognition of such damages and defects is of great importance.

Before these sandwich panels can be implemented as structural components in the field, it is necessary to examine and quantify their sensitivity to imperfections on stiffness and load capacity. ${ }^{12}$ In the early work, Evans et al. ${ }^{13}$ and Wadley et al. ${ }^{5}$ examined the influence of imperfections on the mechanical performance of the truss core. Wallach and Gibson ${ }^{14}$ investigated the effect of randomly distributed defects of a three-dimensional (3D) truss material on Young's modulus and

\footnotetext{
'Key Laboratory for Mechanics in Fluid Solid Coupling Systems, Institute of Mechanics, Chinese Academy of Sciences, Beijing, China

${ }^{2}$ School of Engineering Science, University of Chinese Academy of Sciences, Beijing, China
}

\section{Corresponding author:}

Hongwei Song, Key Laboratory for Mechanics in Fluid Solid Coupling Systems, Institute of Mechanics, Chinese Academy of Sciences, Beijing 100190, China.

Email: songhw@imech.ac.cn 
compressive strength numerically. The modulus and strength decrease linearly with the fraction of members removed. Russell and Hilary ${ }^{12}$ studied the retention of their stiffness and load capacity in the presence of imperfections.

Investigations of the effect of damages and defects on the vibration properties of sandwich structures are also carried out. $\mathrm{Hu}$ and $\mathrm{Hwu}^{15}$ studied the effects of face sheets, core, and delamination on free vibration of composite sandwich plates. Kim and Hwang ${ }^{16}$ analyzed the effect of debonding extent on the flexural stiffness and natural frequency of honeycomb sandwich beam. The flexural stiffness and natural frequency would decrease as the debonding emerges or the extent of debonding increases. Sokolinsky et al. ${ }^{17}$ used the higher order theory approach to analysis the free vibration of sandwich beam with a locally damaged core. It is found that a small local damage would cause significant changes in the natural frequencies and corresponding vibration modes, demonstrating vibration information is an effective tool to assess local damage in sandwich beams. Burlayenko and Sadowski ${ }^{18}$ studied the dynamic behavior of a sandwich plate. The effects of debonding size, location, and type on the modal parameters with different boundary conditions were investigated. Buket and Srinivasa ${ }^{19}$ analyzed the influence of curvature and face/core debonding on the vibration behavior of composite sandwich beams. Results reveal that face/core debonding causes reduction in the natural frequencies, whereas damping loss factor values increase with the presence of debonding. Recently, Lou et al. ${ }^{20}$ studied the effects of local damage on the natural frequencies and the corresponding vibration modes of composite pyramidal truss core sandwich structures by numerical simulation. The structural natural frequencies decrease due to the loss of stiffness caused by the existence of local damage of the truss core, and the influence of local damage becomes more significant with the increase in the damage parameter.

Recently, some works were reported on damage detection methods of sandwich structures. Hu et al. ${ }^{21}$ used strain energy method to detect surface cracks in various composite laminates. Kumar et al. ${ }^{22}$ proposed a modal strain energy method for damage identification of composite sandwich beam, and the method can identify both damage location and damage extent. Tian et al. ${ }^{23}$ used uniform load surface (ULS) curvature to detect the delamination defects of the lattice sandwich plate. Zhu et al. ${ }^{24}$ proposed a non-destructive evaluation (NDE) method for honeycomb sandwich beam, which only utilizes the frequency response function (FRF) measured at one point. Genetic algorithm is used to inverse the damage parameters, and the objective function is set according to the model-based FRF and the experimentally measured FRF. The experimental results demonstrate that the method can inverse the damage location and size with acceptable precision. In the above proposed methods, information of healthy structure must be used as baseline to identify the damage.

However, in the actual evaluation, a baseline in a healthy condition or obtained from a finite element model (FEM) is usually unreliable and may bring large error due to environment, boundary conditions, and so on. ${ }^{25}$ Therefore, baseline-free damage identification methods must be developed for sandwich structures. Based on wavelet transform, Andrzej ${ }^{26}$ proposed a novel approach of two-step damage detection for composite sandwich structures with honeycomb-core. The experimental results show that the proposed method can detect different types of damages successfully. A healthy model is not needed. Based on ULS, a baselinefree NDE method is developed to determine the debonding of truss bar in composite lattice truss core sandwich structures. ${ }^{25}$ The method is proposed according to the synergy of gapped smoothing method (GSM) and Teager energy operator (TEO), and is denoted as GSM-TEO method. The weight of low-order modes in ULS is much larger than high-order modes. However, in practical applications, the effect of damage with small extent on low-order modes is very limited. Therefore, the methods based on ULS are not effective to identify damage with small extent, especially in the case of multiple damages including slight ones.

Although much effort has been devoted to explore the behavior of the SPTCs, little research has been undertaken into NDE and health control of this new structure. $^{25}$

In this study, a baseline-free NDE method is proposed for metallic SPTCs. Damage index $D I_{m}$ based on the flexibility matrix and GSM method is presented, and $m$ is a weight coefficient to consider effects of damages on both low- and high-order modes. Numerical simulations and experimental validations of metallic pyramidal SPTC with single and multiple damages are conducted. Comparisons of the present method and GSM-TEO method ${ }^{25}$ are conducted. Moreover, parameter $D I_{m}^{*}$ is also defined by processing $D I_{m}$ with TEO, and comparisons of $D I_{m}$ and $D I_{m}^{*}$ for damage identification of metallic SPTCs are also carried out.

\section{Formulation of damage identification method}

The flexibility matrix $F$ of a structure can be expressed as follows

$$
F=\sum_{k=1}^{n} \frac{\Phi_{k} \Phi_{k}^{T}}{\omega_{k}^{2}}
$$




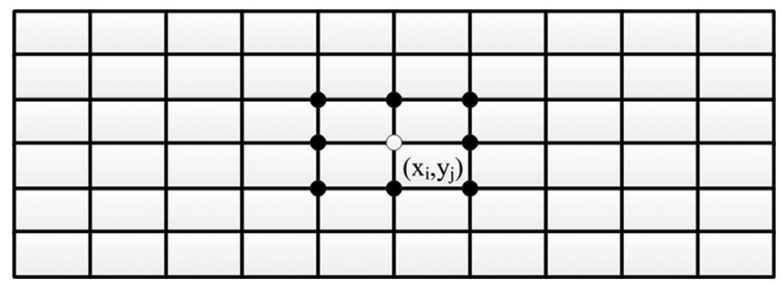

Figure I. Gapped grid points.

where $\omega_{k}$ and $\Phi_{k}$ are the $k$ th order of natural frequency and mass normalized mode, respectively, $n$ is the number of degrees of freedom.

In the flexibility matrix $F$, all order modes are included. To consider only one order mode, the $k$ th component $F_{k}$ of the flexibility matrix $F$ is obtained as equation (2). Then, the $k$ th component of modal deflection at point $i$ under uniform unit load all over the structure can be expressed as equation (3)

$$
\begin{gathered}
F_{k}=\frac{\Phi_{k} \Phi_{k}^{T}}{\omega_{k}^{2}} \\
D_{k}(i)=\sum_{j=1}^{n} \frac{\Phi_{k}(i) \Phi_{k}(j)}{\omega_{k}^{2}}=\frac{\Phi_{k}(i) \sum_{j=1}^{n} \Phi_{k}(j)}{\omega_{k}^{2}}
\end{gathered}
$$

The $k$ th component of modal deflection $M D C_{k}$ can be deduced as equation (4), and it only relates with the $k$ th-order mode. Besides, from equation (4), it is also seen $M D C_{k}$ is linear with $\omega_{k}^{-2}$

$$
M D C_{k}=\left\{D_{k}(i)\right\}=F_{k} \cdot I
$$

where $I$ is $\{1, \ldots, 1\}_{1 \times n}^{T}$.

$\mathrm{GSM}^{27,28}$ is proposed to overcome the problem damage identification without a baseline or healthy data. In this study, GSM is employed. Eight neighboring points are selected to fit the value of point $\left(x_{i}, y_{j}\right)$, as shown in Figure 1. By cubic polynomial, the estimated $M D C_{k}\left(x_{i}, y_{j}\right)^{\prime}$ could be expressed as follows

$$
\begin{aligned}
M D C_{k}\left(x_{i}, y_{j}\right)^{\prime}= & a_{0}+a_{1} x_{i}+a_{2} y_{j} \\
& +a_{3} x_{i} y_{j}+a_{4} x_{i}^{2}+a_{5} y_{j}^{2}+a_{6} x_{i}^{2} y_{j}+a_{7} x_{i} y_{j}^{2}
\end{aligned}
$$

where the eight coefficients $a_{0}$ to $a_{7}$ could be obtained by the eight points around point $\left(x_{i}, y_{j}\right)$, and are expressed as follows

$$
A\left(x_{i}, y_{j}\right)^{k}=C\left(x_{i}, y_{j}\right) \times M D C\left(x_{i}, y_{j}\right)^{K}
$$

where $A\left(x_{i}, y_{j}\right)^{k}$ is $\left\{a_{0}, a_{1}, \ldots, a_{6}, a_{7}\right\}_{1 \times 8}^{\mathrm{T}}$ for point $\left(x_{i}, y_{j}\right)$ in $k$ th-order mode, $\operatorname{MDC}\left(x_{i}, y_{i}\right)^{k}$ is $\left\{M D C_{k}\left(x_{i-1}, y_{j-1}\right)\right.$,
$M D C_{k}\left(x_{i-1}, y_{j}\right), \ldots, M D C_{k}\left(x_{i+1}, y_{j}\right)$,

$\left.\operatorname{MDC}_{k}\left(x_{i+1}, y_{j+1}\right)\right\}_{1 \times 8}^{\mathrm{T}} . C\left(x_{i}, y_{j}\right)$ is the coefficient matrix

$$
C\left(x_{i}, y_{j}\right)=\left[\begin{array}{ccccc}
\mathbf{1} & x_{i-1} & \cdots & x_{i-1}^{2} y_{j-1} & x_{i-1} y_{j-1}^{2} \\
\mathbf{1} & x_{i-1} & \cdots & x_{i-1}^{2} y_{j} & x_{i-1} y_{j}^{2} \\
\vdots & \vdots & \ddots & \vdots & \vdots \\
\mathbf{1} & x_{i+1} & \cdots & x_{i+1}^{2} y_{j} & x_{i+1} y_{j}^{2} \\
\mathbf{1} & x_{i+1} & \cdots & x_{i+1}^{2} y_{j+1} & x_{i+1} y_{j+1}^{2}
\end{array}\right]^{-1}
$$

Because $M D C_{k}$ is linear with $\omega_{k}^{-2}$ and the coefficient matrix $C\left(x_{i}, y_{j}\right)$ is independent of $\omega_{k}^{-2}$, the relationship between the eight coefficients $A\left(x_{i}, y_{j}\right)^{k}$ and $\omega_{k}^{-2}$ is linear. Therefore, the estimated value $M_{D} C_{k}\left(x_{i}, y_{j}\right)^{\prime}$ is also linear with $\omega_{k}^{-2}$.

Based on the real value $M D C_{k}\left(x_{i}, y_{j}\right)$ and the estimated value $M D C_{k}\left(x_{i}, y_{j}\right)^{\prime}$, the differential value $Z_{k}$ of point $\left(x_{i}, y_{j}\right)$ can be expressed as equation (8)

$$
Z_{k}\left(x_{i}, y_{j}\right)=\frac{\left(M D C_{k}\left(x_{i}, y_{j}\right)-M D C_{k}\left(x_{i}, y_{j}\right)^{\prime}\right)^{2}}{\sum_{i=1}^{F} \sum_{j=1}^{F}\left(M D C_{k}\left(x_{i}, y_{j}\right)-M D C_{k}\left(x_{i}, y_{j}\right)^{\prime}\right)^{2}}
$$

where $E$ and $F$ are the number of columns and lines of the measuring points, respectively.

Because both $M D C_{k}\left(x_{i}, y_{j}\right)$ and $M D C_{k}\left(x_{i}, y_{j}\right)^{\prime}$ are linear with $\omega_{k}^{-2}, Z_{k}$ is independent of natural frequency $\omega_{k}$. From equation (8), it is also seen that $Z_{k}$ is only related with the $k$ th-order mode.

As we know, influences of different damages on different order modes are quite divergent. Some damages have great influences on low-order modes and some have great influences on high-order modes. The diversity of sensitiveness is especially obvious for specimens with multiple damages. Therefore, to identify various damages, the damage index $D I_{m}$ is defined

$$
D I_{m}=\sum_{k=K_{1}}^{K_{2}} \alpha_{k}^{m} Z_{k}
$$

where $K_{1}$ and $K_{2}$ are the beginning and the ending order modes in the practical case. $\alpha_{k}^{m}$ is defined as the weight coefficient of the $k$ th component

$$
\alpha_{k}^{m}=\frac{\omega_{k}^{m}}{\sum_{k=K_{1}}^{K_{2}} \omega_{k}^{m}}
$$

$\alpha_{k}^{m}$ could determine the weights of low-order modes and high-order modes in $D I_{m}$, and $m$ is an integer. When parameter $m$ takes different values, the relationship between $k$ and $\alpha_{k}^{m}$ would expressed as follows 
when $m<0, k$ increases $\rightarrow \alpha_{k}^{m}$ decreases

when $m=0, k$ increases $\rightarrow \alpha_{k}^{0}=\frac{1}{K_{2}-K_{1}}$

when $m>0, k$ increases $\rightarrow \alpha_{k}^{m}$ increases

When $m$ is less than 0 , the weight of low-order modes in $D I_{m}$ is higher than high-order modes. When $m$ is equal to 0 , the weight of low-order modes in $D I_{m}$ is equal to high-order modes. When $m$ is larger than 0 , the weight of low-order modes in $D I_{m}$ is smaller than high-order modes. Therefore, damage index $D I_{-2}, D I_{0}$, and $D I_{2}$ are selected to identify damages of SPTCs in the article.

\section{Numerical simulation}

\section{Numerical model}

Figure 2 gives the sketch of the pyramidal SPTC model being simulated. The unit cell of pyramidal truss is shown in Figure 2(a), where $h_{c}=8 \mathrm{~mm}, t_{c}=1 \mathrm{~mm}$, $\theta=45^{\circ}$. Figure 2(b) shows that there are 10 units along the length and width direction of the panel, respectively. The thickness of the face sheets is $1 \mathrm{~mm}$. The SPTC is made of stainless steel with Young's

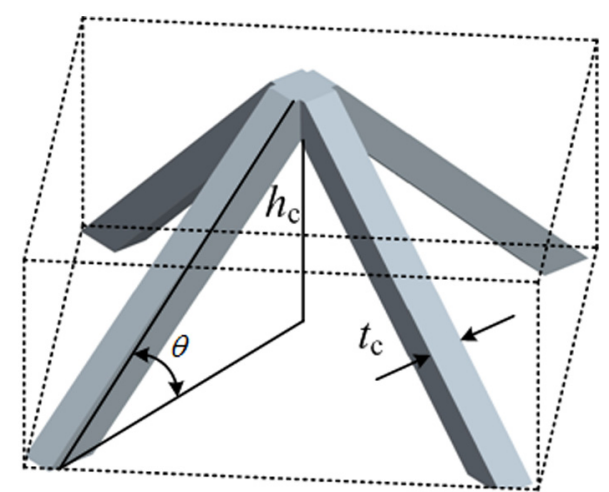

(a)

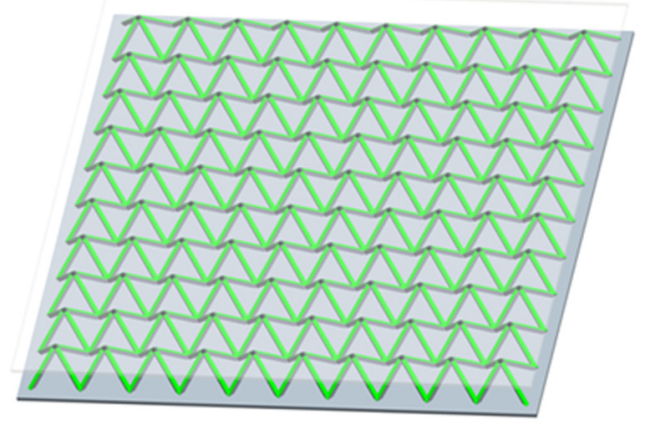

(b)

Figure 2. Model of SPTC: (a) unit cell of pyramidal truss and (b) the sandwich panel with pyramidal truss core. modulus of $200 \mathrm{GPa}$, Poisson ratio of 0.3 , and mass density of $7800 \mathrm{~kg} / \mathrm{m}^{3}$. "Beam189" and "Shell181" are selected for the truss bars and the face sheets in the numerical model. The boundary condition for the SPTC is fully clamped (CCCC). In all, 10 cases including single damage, multiple damages of the same extent, and multiple damages of different extent are investigated, and damaged specimens are shown in Figure 3. The meaning of each damage case will be detailed in sections "Single damage identification," "Multiple damages identification," and "Effect of damage location and damage extent."

To demonstrate the role of weight coefficient $m$, the first 20 modes are used to calculate damage index $D I_{m}$ in the simulations. It is worth mentioning that the number of selected modes for calculating $D I_{m}$ is determined by the practical situation and only a reasonable number of modes would be selected in actual experiments.

\section{Effect of damages on natural frequencies}

Damage index $\eta_{i}$ (equation (12)) is defined to evaluate the effect of damages on natural frequencies

$$
\eta_{i}=\frac{\left|f_{i}^{D}-f_{i}^{I}\right|}{f_{i}^{I}}
$$

where $f_{i}^{D}$ is the $i$ th natural frequency of the damaged model, and $f_{i}^{I}$ is the $i$ th natural frequency of the intact model. The results of $\eta_{i}$ for the first 20 order modes are shown in Figure 4.

As we know, higher order modes are normally more sensitive to damages than lower order modes, and the 10 curves in Figure 4 could basically demonstrate this tendency. However, many factors could influence the sensitivity of some order modes to damages, such as the constraint condition, damage location, damage types, damage extent, and the mode shape. Therefore, some relative low-order mode (like 14th) would be more sensitive to some damage cases (SD3, MD2, MD3, MD4, MD5, MD6, and MD7) than higher order modes (like 15th-20th). When the damage information is unknown, both low-order modes and high-order modes should be used for various damages identification.

\section{Single damage identification}

Single damage identification with different extent was conducted by numerical simulation. Damage cases with $1 / 2$ cell missing, $3 / 4$ cell missing, and whole cell missing are investigated, named SD1, SD2, and SD3, respectively (as shown in Figure 3(a) to (c)). $D I_{m}$ results of the three cases are shown in Figure 5(a) to (c). And the first 20 curvature modes of SD1 are also presented in Figure 6. 

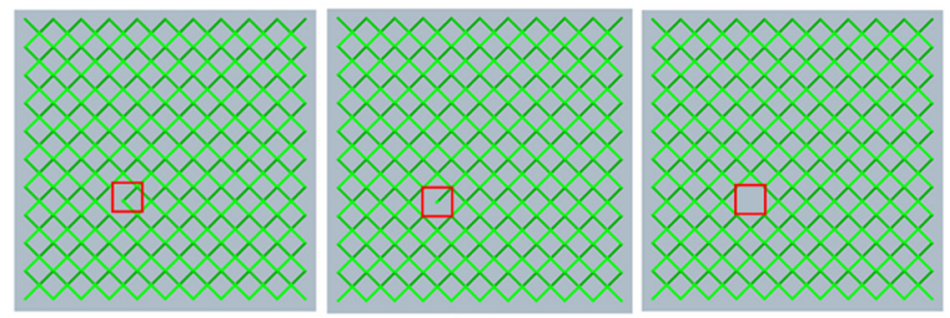

(a)
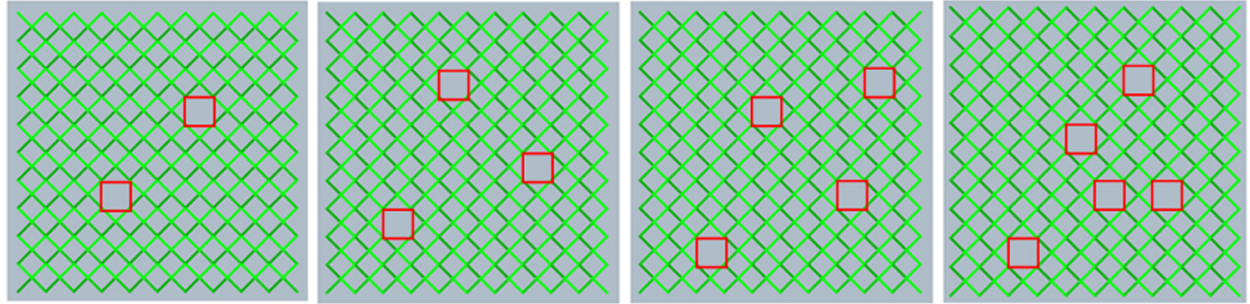

(b)
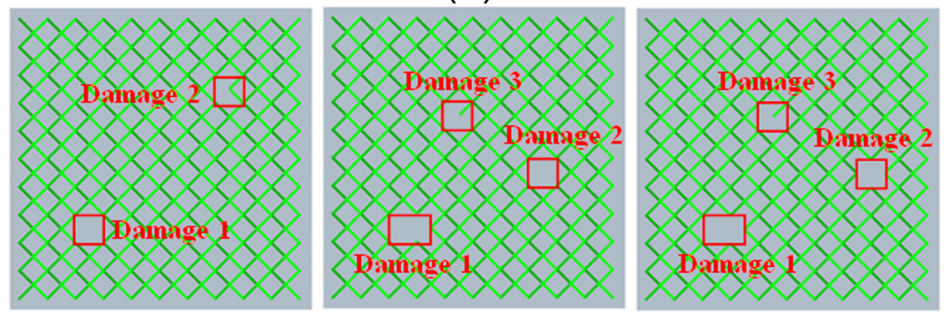

(c)

Figure 3. Ten types of damages. (a) single damage: SDI, SD2, and SD3. (b) multiple damages: MDI, MD2, MD3, and MD4. (c) multiple damages of different extent: MD5, MD6, and MD7.

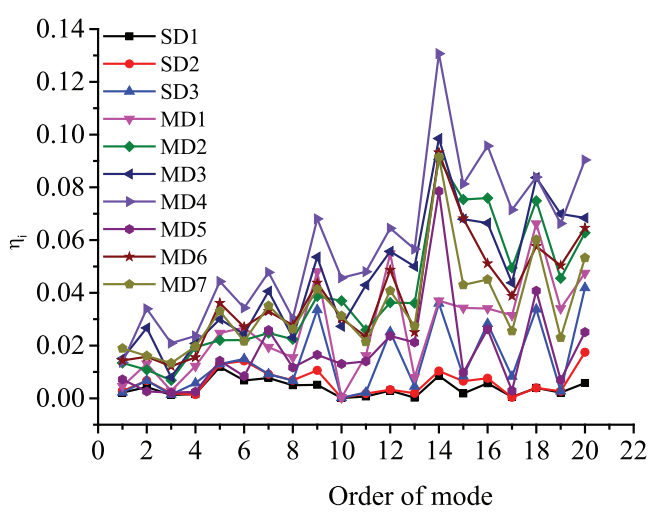

Figure 4. Effect of damages on natural frequencies.

Figure 6 reveals that the singularity caused by the contact nodes between the truss bars and face sheet is so large that the actual damage is shielded. However, based on the proposed parameter $D I_{m}$, the single damage location could be highlighted effectively as the red arrows pointed in Figure 5(a) although there are peaks at the contact nodes, and the fluctuations at the four edges are also very obvious. It demonstrates the damage index $D I_{m}$ is good at suppressing the effect of the contact nodes for pyramidal SPTCs.

According to $D I_{-2}, D I_{0}$, and $D I_{2}$ results in Figure 5, it is observed that the fluctuations caused by boundary conditions and contact nodes become small as the weight of high-order modes increases. This is because the local vibration modes at damage locations are more easily to be excited in higher order modes. Therefore, the fluctuations at the four edges and the contact nodes are suppressed more effectively. Meanwhile, as the number of missing bars increases (SD1-SD3), the singularity at the damage location becomes more obvious. It demonstrates the proposed index $D I_{m}$ is sensitive to the damage extent.

\section{Multiple damages identification}

To evaluate the effectiveness of the proposed method for multiple damages identification, pyramidal SPTCs with 2, 3, 4, and 5 damages (named MD1, MD2, MD3, and MD4) are investigated. The distribution of the damages is random, and the extents of all damages are same (whole cell missing). It is observed from Figure 7 (a) to (d) that all the damage locations of the 

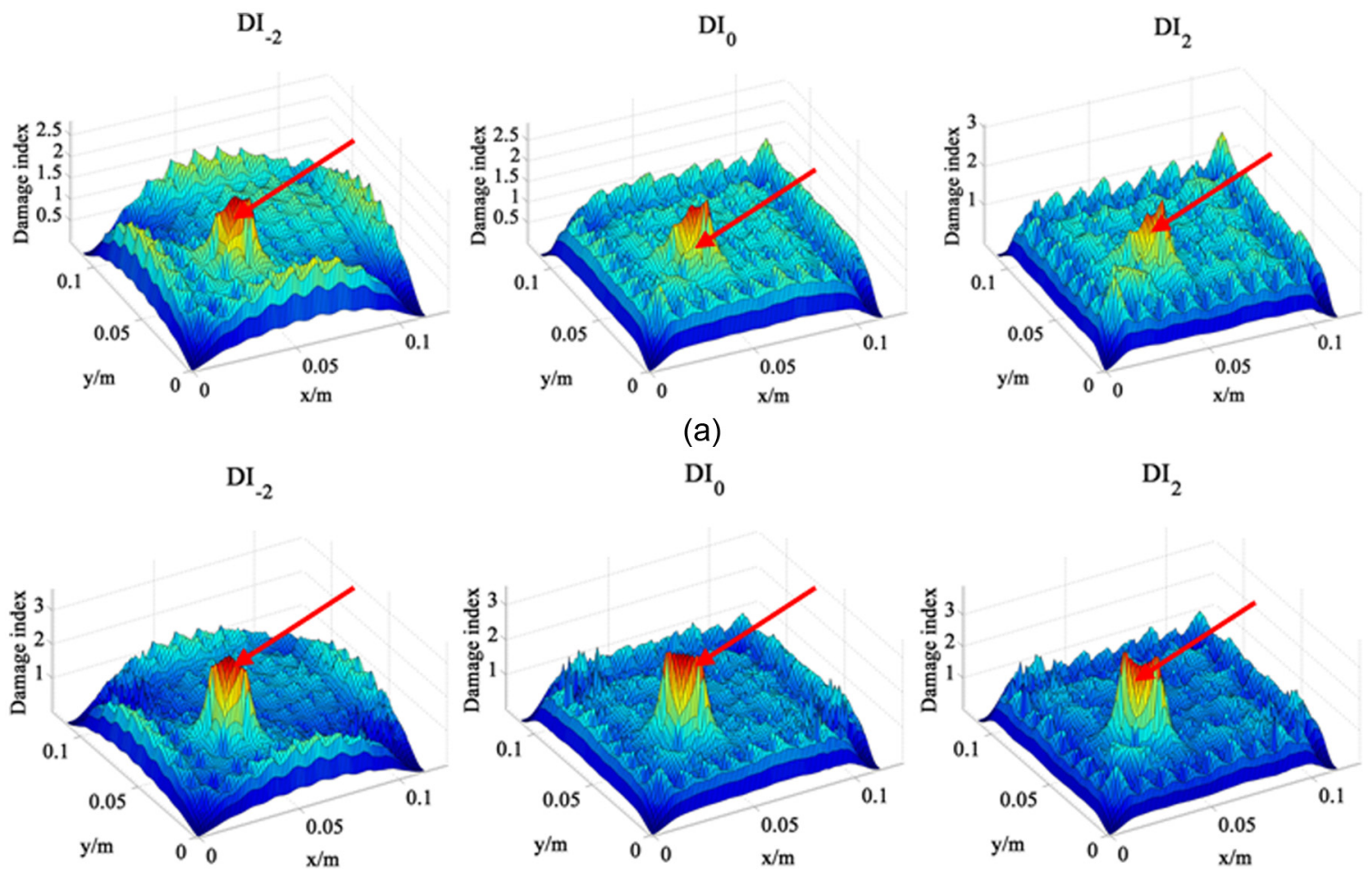

(b)
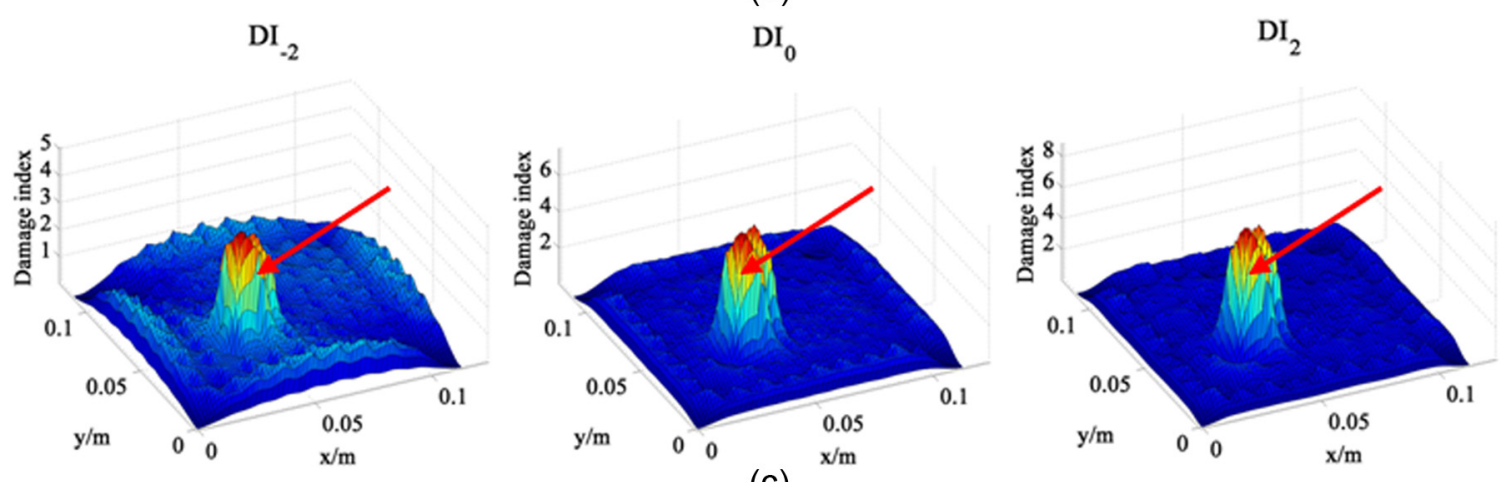

Figure 5. $D I_{m}$ results: (a) SDI, (b) SD2, and (c) SD3.

pyramidal SPTC could be detected, demonstrating the effectiveness of the present method to the randomly distributed multiple damages.

As shown in Figure 7(a), when the weight of loworder modes is high $\left(D I_{-2}\right)$, the singularity caused by the contact nodes is very obvious. As the weight of high-order modes increases, the influence of constraint condition and contact nodes decreases and the two damage locations could be highlighted more effectively. The same conclusions could be obtained from Figure 7(b) to (d). The conclusion is in accordance with single damage cases (section "Single damage identification"). Therefore, increasing the weight of high-order modes is good for suppressing the singularity caused by the contact nodes for both single damage identification and multiple damages identification with the same extent.

Meanwhile, the fluctuations at the four edges and the peaks at the contact nodes become invisible as the number of damages increases, for example, $\mathrm{DI}_{-2}$ results from Figure 7(a) to (d). This is because the local vibrations at multiple damage locations suppress the fluctuations and the peaks. It is displayed that the influence of the fluctuations and the peaks to damage detection would decrease as the number of damages increases for pyramidal SPTCs. 

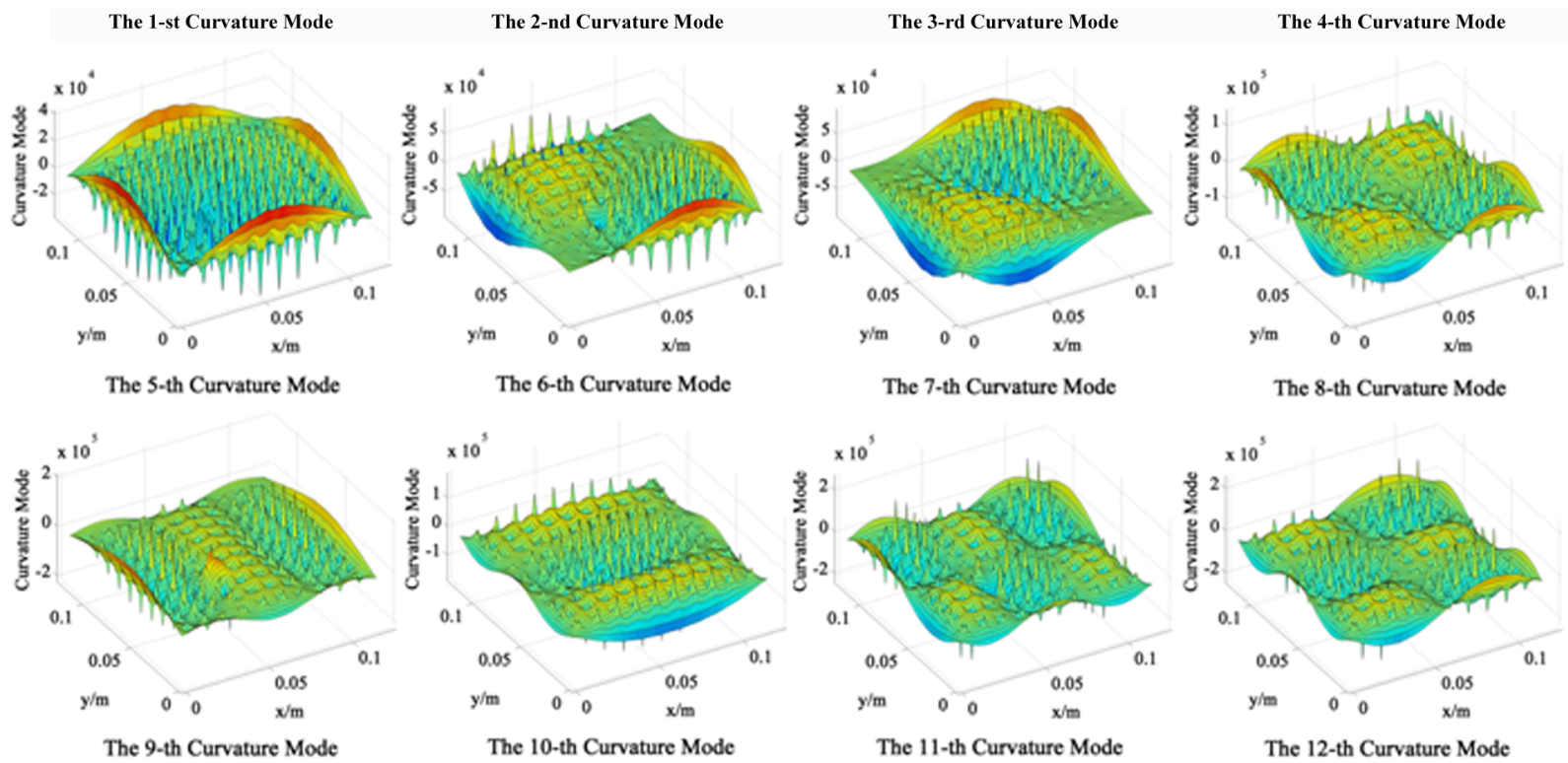

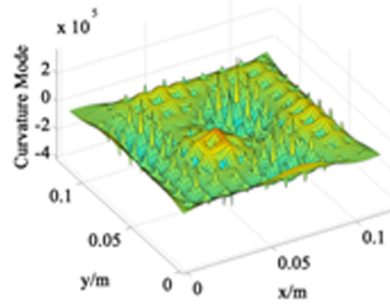

The 13-th Curvature Mode

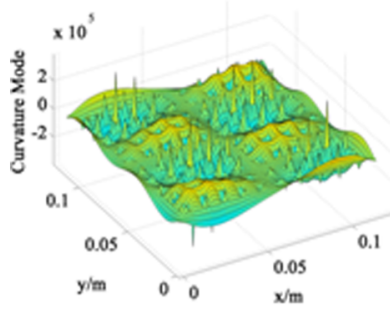

The 17-th Curvature Mode

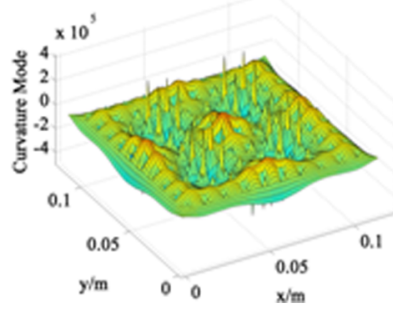

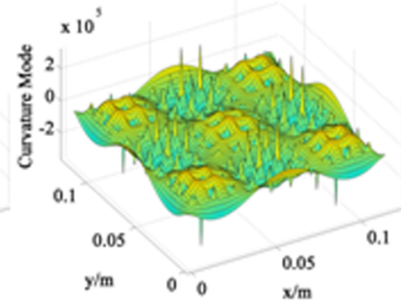

The 15-th Curvature Mode

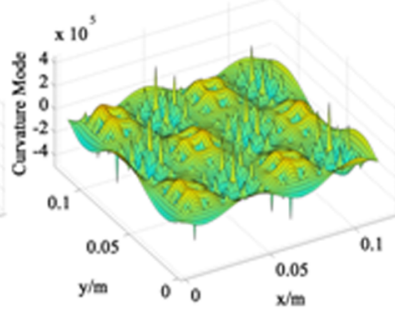

The 19-th Curvature Mode

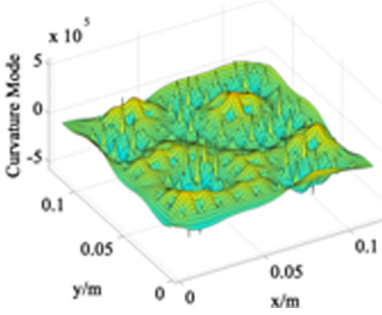

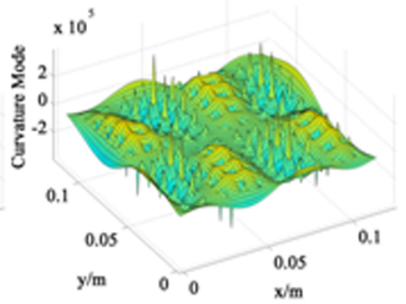

The 16-th Curvature Mode

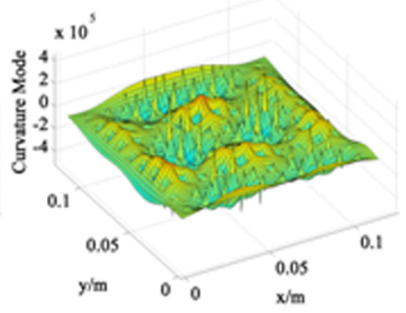

The 20-th Curvature Mode

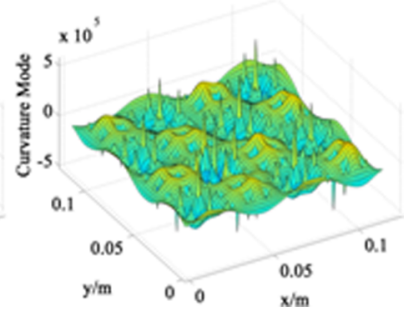

Figure 6. The first 20 curvature modes of SDI.

\section{Effect of damage location and damage extent}

Multiple damage cases with different extent are employed in this section to investigate the effect of damage location and damage extent on modal variation. The sketches of MD5, MD6, and MD7 are shown in Figure 3(c).
As shown in Figure 8(a), the two damages of different extent could be identified based on $\mathrm{DI}_{-2}$. It is also found that the peak caused by damage 1 is more obvious than damage 2, which is in accordance with the MD5 damage style. However, as the weight of highorder modes increases, damage 2 becomes more 


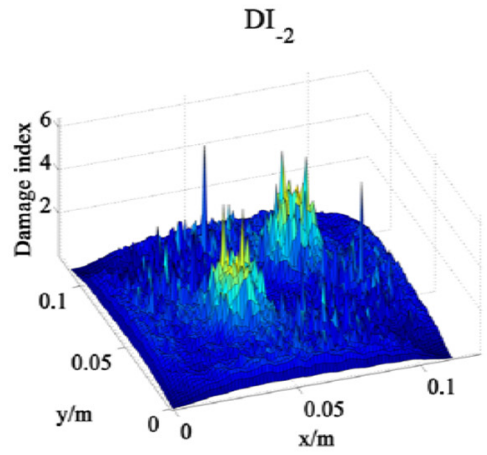

$\mathrm{DI}_{-2}$

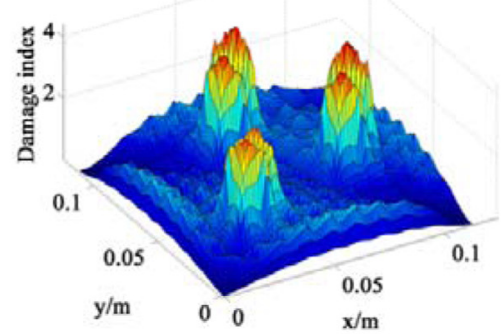

$\mathrm{DI}_{-2}$

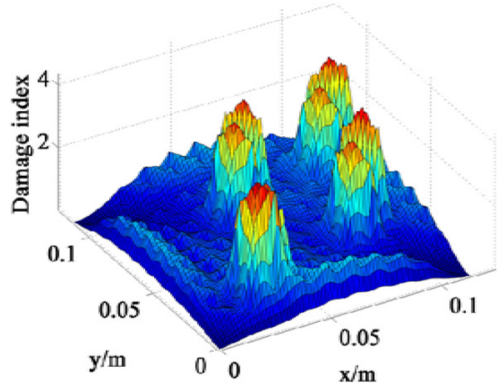

DI $_{-2}$

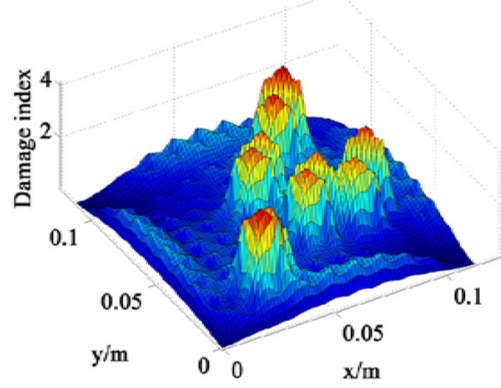

$\mathrm{DI}_{0}$

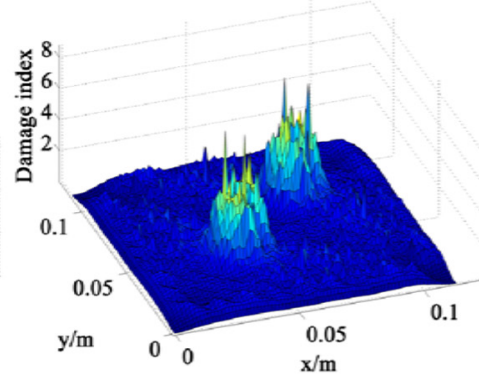

(a)

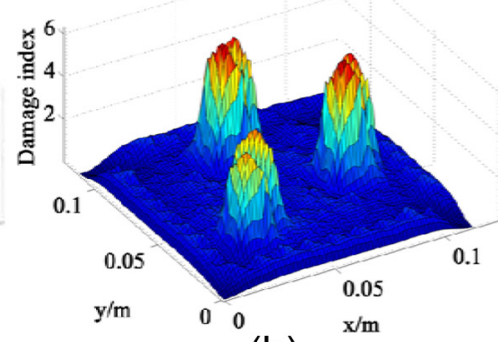

(b)

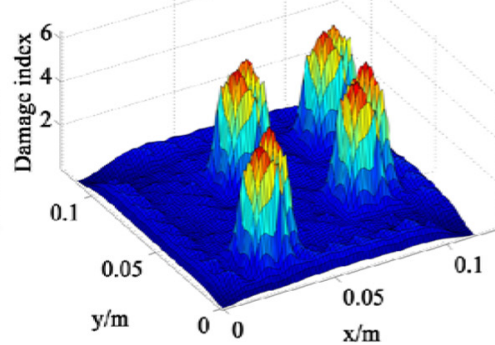

(c)

DI

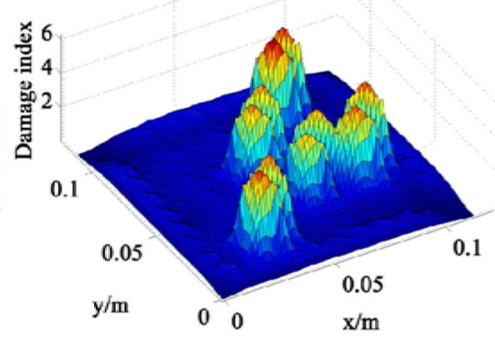

(d)

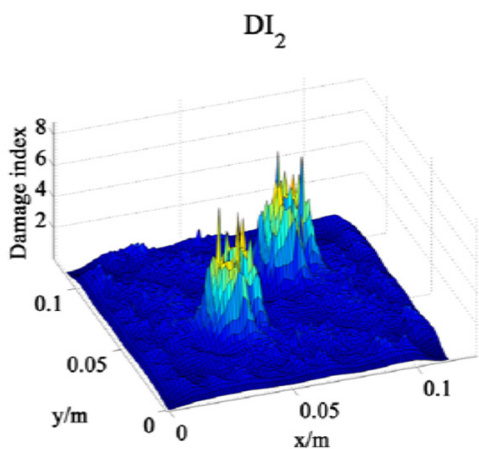

$\mathrm{DI}_{2}$

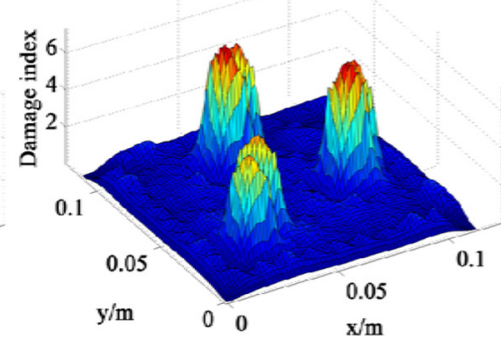

$\mathrm{DI}_{2}$

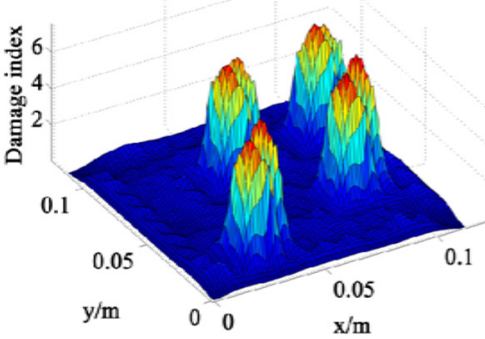

$\mathrm{DI}_{2}$

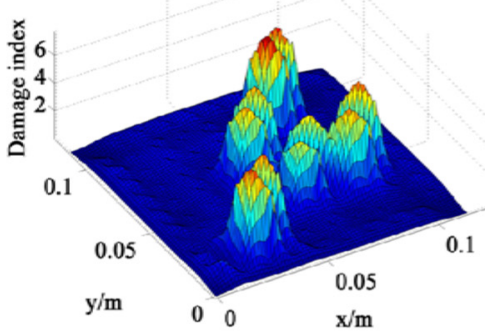

Figure 7. $D I_{m}$ results: (a) MDI, (b) MD2, (c) MD3, and (d) MD4.

invisible and only damage 1 could be clearly detected, see $\mathrm{DI}_{2}$ result in Figure 8(a). This is due to the singularity caused by main damage (damage 1 ), which is more serious than slight damage (damage 2) in high-order modes. When the weight of high-order modes is larger than low-order modes, the slight damage is shielded. 


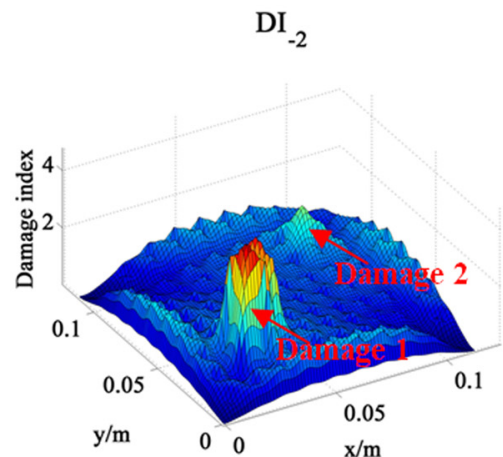

$\mathrm{DI}_{-2}$

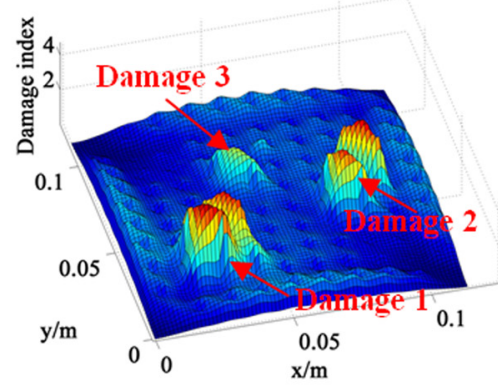

$\mathrm{DI}_{-2}$

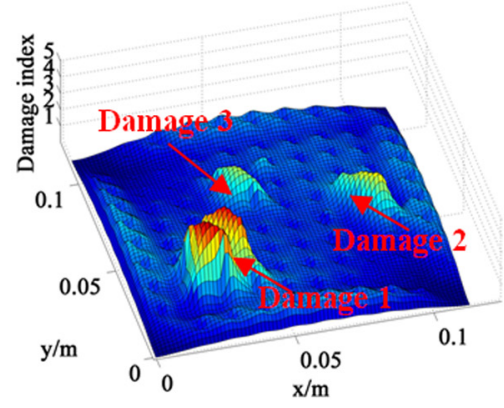

Figure 8. $D I_{m}$ results: (a) MD5, (b) MD6, and (c) MD7.

Therefore, damage index of both low-order modes and high-order modes should be used for multiple damages identification with different extent.

It is observed from Figure 8(b) that all the three damages are located accurately. Although the extent of damage 1 (six bars or one half-cell missing) is more serious than damage 2 (four bars or one cell missing), the peaks are almost the same, because the location of damage may also influence the vibration modes greatly. The damage extent could not be identified only according to the value of $D I_{m}$. Therefore, when multiply damages of different extents exist, more precise damage extent identification method needs to be developed for the pyramidal SPTCs.

Comparing Figure 8(b) with Figure 8(c), it is observed that the peak at damage 2 of MD7 is much smaller than that of MD6 although both damages miss the same number of bars. However, the four missing bars of damage 2 in MD6 belong to one unit cell, whereas the four missing bars of damage 2 in MD7 belong to the adjacent two unit cells. There are still two residual bars to connect the face sheet, respectively, for each of the two half-cell missing damage in MD7. It demonstrates the effect of the whole truss core missing 


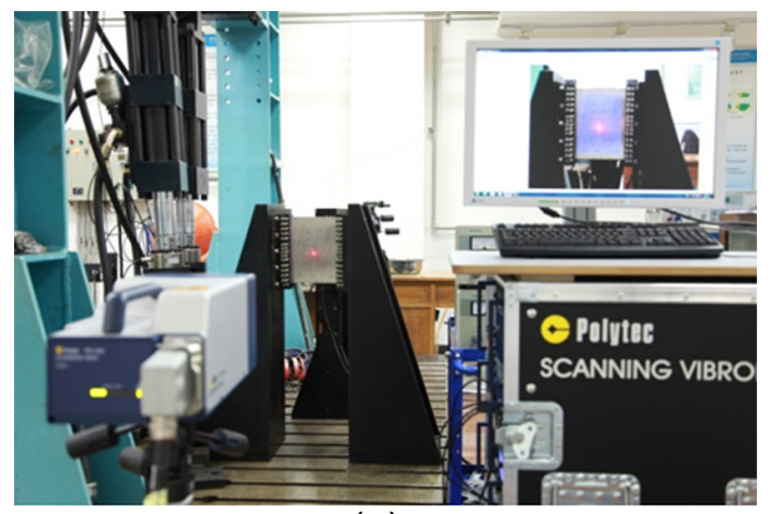

(a)

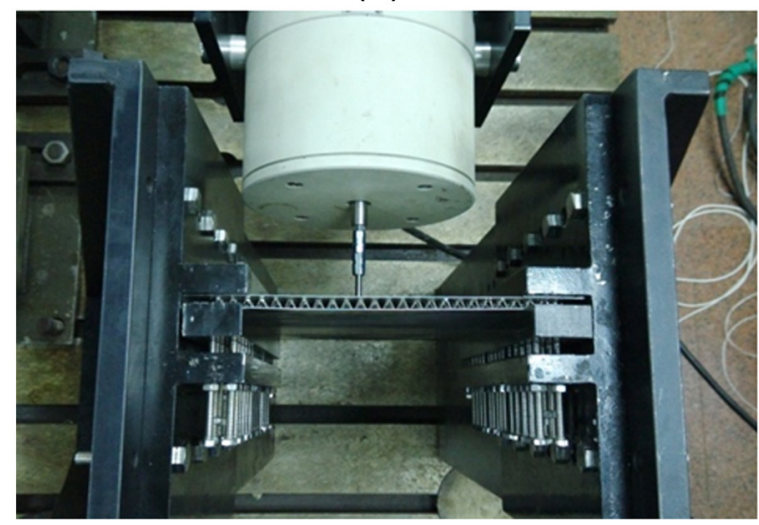

(b)

Figure 9. Experimental setup: (a) front view and (b) top view.

on vibration modes is much larger than two half-cell missing.

\section{Experimental validation}

In this section, vibration experiments of metallic SPTCs are conducted, and the experimental results are utilized to validate the present damage identification method.

\section{Experimental setup}

In the experiment, the specimens of metallic SPTC with pre-fabricated damage are excited by a vibrator (JZK50), and the experimental setup is shown in Figure 9(a). Two edges of the specimens are clamped. The excitation signal is generated by the control system of a laser Doppler vibrometer (Polytec, PSV-400) and amplified by a power amplifier (YE5874A) before input to the shaker. The frequency bandwidth of the excitation signal was set in the range of $0-3 \mathrm{kHz}$ with the resolution of $1 \mathrm{~Hz}$.

The laser Doppler vibrometer is also used to measure the structural vibration response and the scanning points are focused on the front surface of the face sheet,

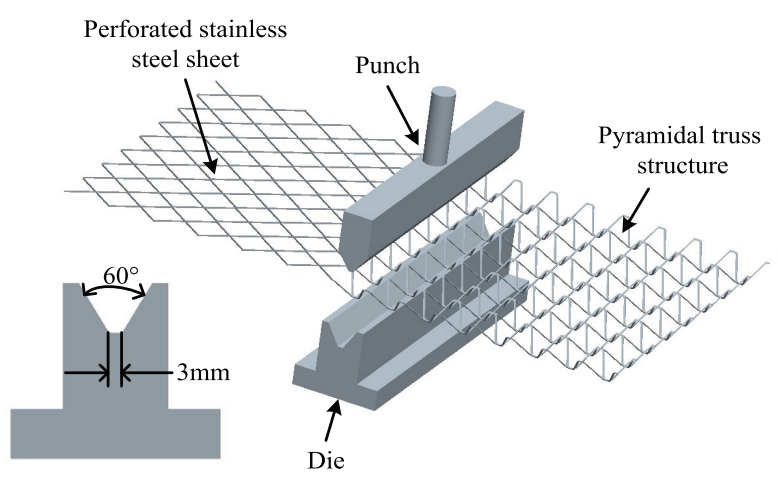

Figure 10. The punching operation for manufacturing the pyramidal truss cores.

and the excitation is on the other side of the face sheet. The obtained excitation and structural response signal are used to calculate the FRF to get the structural modal information. In order to obtain good quality of modal information, the FRF for each scanning point was averaged three times in each test.

\section{Fabrication of specimen}

Up to now, the fabrication of a SPTC is still in the laboratory-level stage. In this work, the metallic pyramidal truss cores with a relative density $\rho$ of about $3 \%$ are fabricated from $0.7 \mathrm{~mm}$ thick perforated stainless steel sheet by folding technique. Figure 10 shows the sketch of the punching operation to fold the perforated sheet into pyramidal truss cores, using a punch-and-die pair of $60^{\circ}$ angle. The punch-and-die pair is designed with a $3 \mathrm{~mm}$ terrace to obtain flat areas at nodal regions, in order to avoid node fracture and enlarge the joint area between truss cores and face sheets. The brazing technique, which leaves no flaw on the face sheets, is utilized to join the truss core with face sheets. The thickness of face sheet is $0.9 \mathrm{~mm}$ and the thickness of the truss core is $7 \mathrm{~mm}$. The dimension of the metallic SPTC specimen is $250 \mathrm{~mm} \times 250 \mathrm{~mm}$, and there are 22 cells in the row and 15 cells in the column.

Compared with composite SPTCs recently being tested, for example, the specimen used in Li's work (2015), the dimensions of truss core cell of metallic SPTCs fabricated in this study are much smaller. Meanwhile, in the study, the four bars in each cell share the same node that is connected to the face sheet. Therefore, damages in the present metallic SPTCs are more difficult to be identified than composite SPTCs.

In the study, cell missing damages are used to simulate structural damage. The truss core cells of the damage location are cut out before brazing. Two damage styles are fabricated in the work. The first damage style 


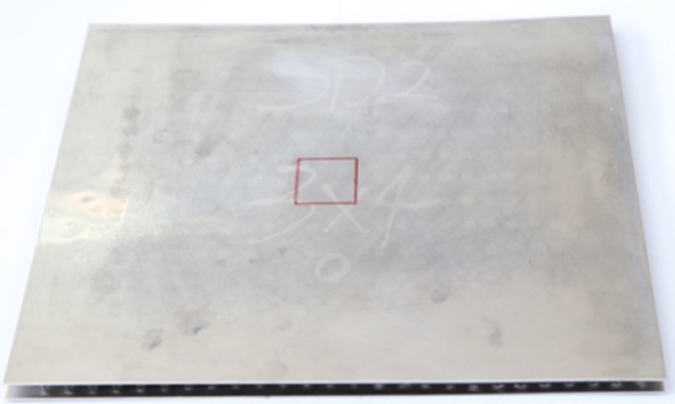

(a)

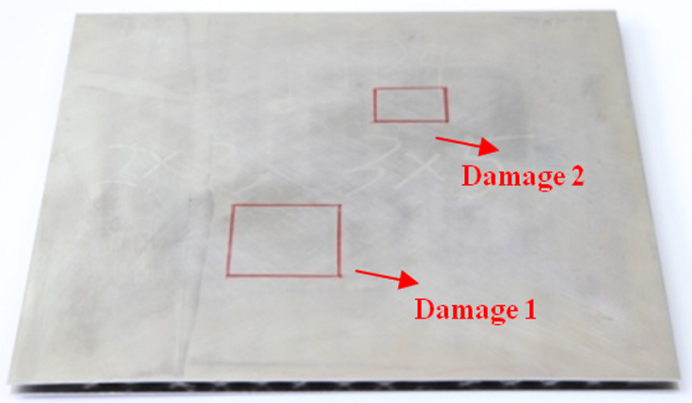

(b)

Figure I I. Damaged specimens: (a) single damage, SD and (b) double damages, DD.

is single damage, named SD (Figure 11(a)). The red rectangle zone is the damage location and region, and the size of the damage is $3 \times 4$ cells missing. The second damage style is double damages with different extents, named DD (Figure 11(b)). The size of damage 1 is $3 \times 5$ cells missing, and the size of damage 2 is $2 \times 3$ cells missing.

\section{Experimental results}

The scanning points of SD specimen and DD specimen are shown in Figure 12, and the damages are highlighted as the red rectangle zones. Based on the measuring results, 4 and 7 modes were used to calculate $D I_{m}$ for SD and DD specimens, respectively, with results shown in Figure 13. It is observed from Figure 13(a) that the single damage location could be identified effectively by parameter $D I_{m}$. When the weight of loworder modes is equal to high-order modes, fluctuations at the two edges of free boundary are obvious. However, the damage still could be located accurately by combining $D I_{-2}, D I_{0}$, and $D I_{2}$.

According to $D I_{0}$ and $D I_{2}$ results in Figure 13(b), it is seen that both damage 1 and damage 2 can be identified. It is also found that the peak caused by damage 1

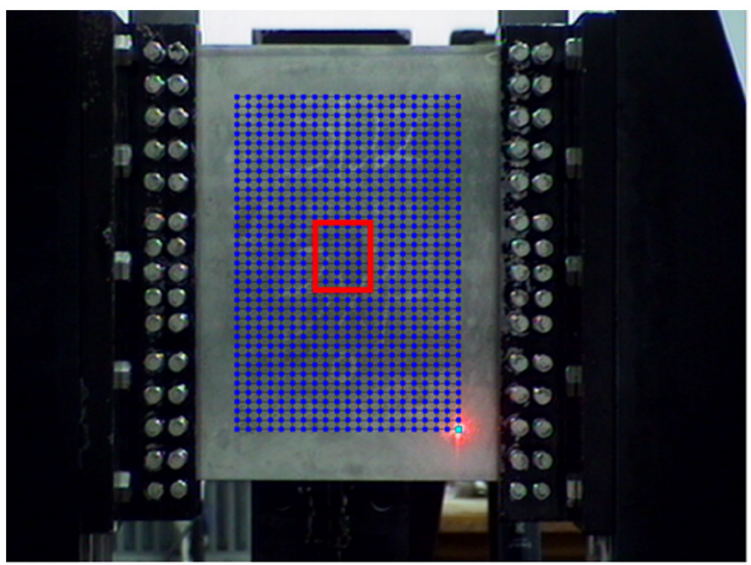

(a)

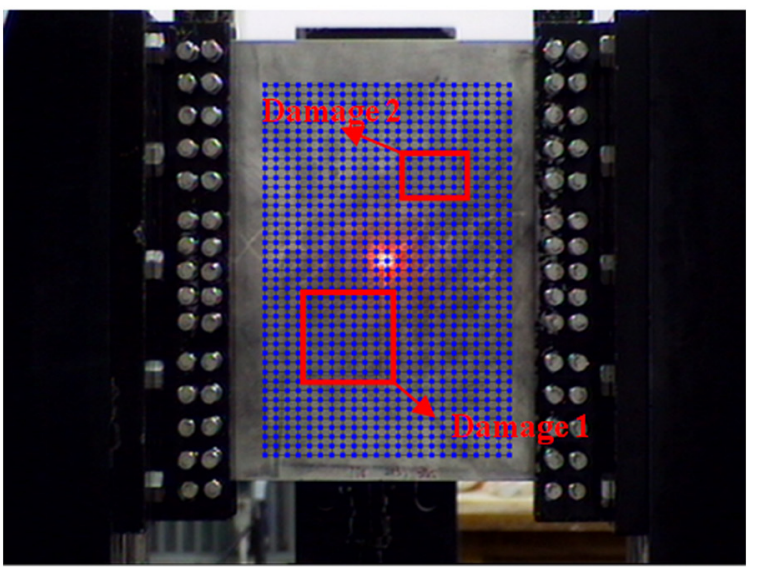

(b)

Figure I2. Scanning points of the specimen: (a) SD specimen and (b) DD specimen.

is more obvious than damage 2 , which is in accordance with the damage style of the DD specimen. However, only damage 1 could be identified according to $\mathrm{DI}_{-2}$ results. Because local vibrations at damage 2 are not excited in first several order modes. Therefore, if only low-order modes are used, damage 2 would be shielded and not be identified. Meanwhile, the singularity caused by damage 2 becomes relatively weak as the weight of high-order modes increases. Because the extent of damage 1 is more serious than damage 2 , the singularity of high-order modes caused by damage 1 is much larger than damage 2 as the order of mode increases. This conclusion is in accordance with the simulation results of MD5 case. It demonstrates that the weight coefficient $m$ plays an important role for multiple damages identification. The information of both low-order modes and high-order modes should be considered for multiple damages identification. $D I_{m}$ results in case of different $m$ should be combined together for damage identification. 


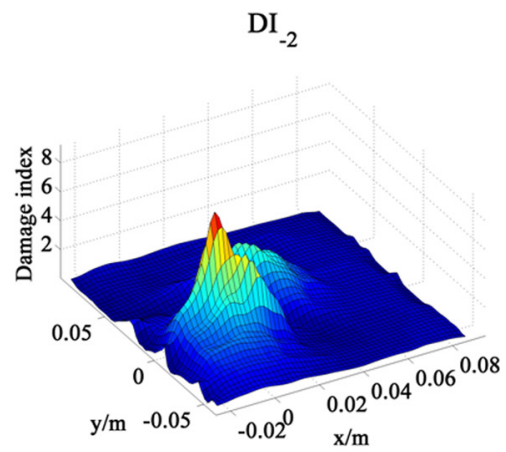

DI $_{-2}$

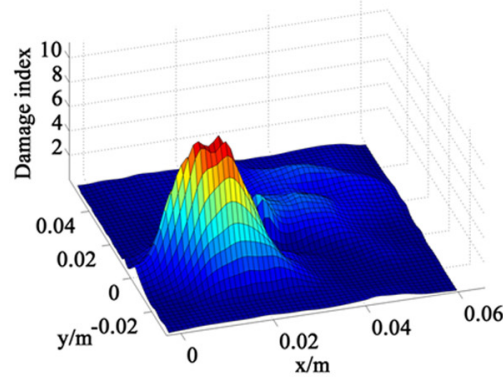

(b)
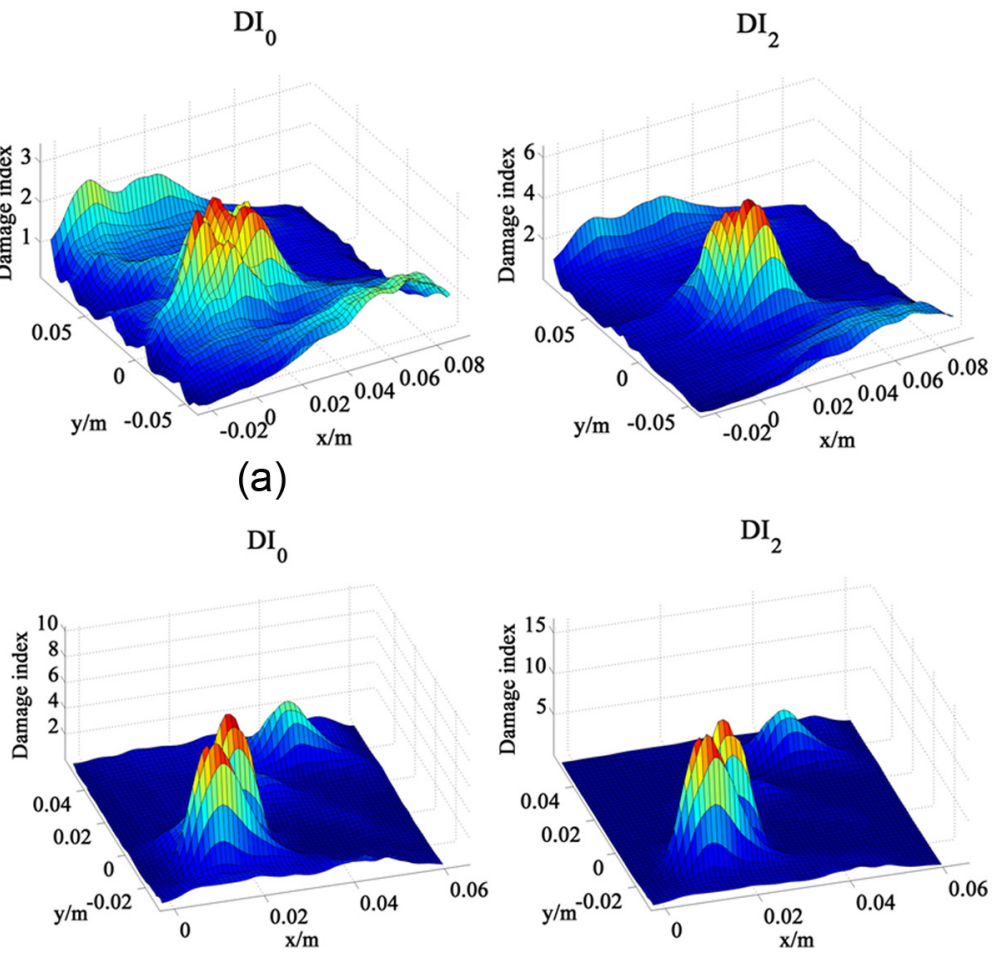

(a)

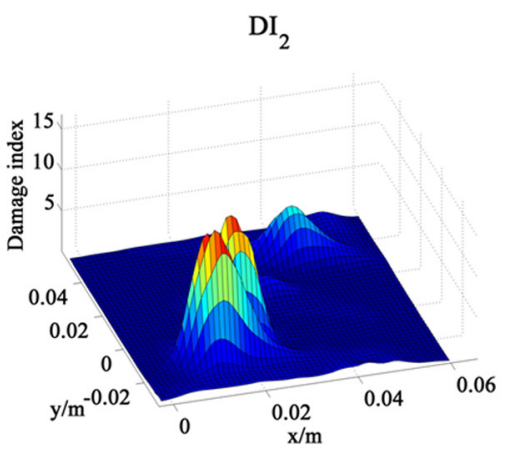

Figure 13. $D I_{m}$ results: (a) SD specimen and (b) DD specimen.

\section{Discussions}

\section{Application of TEO in the present method}

From the above simulation and experimental results, it is seen that the proposed damage index $D I_{m}$ is effective for damage identification of metallic SPTCs. However, the fluctuations caused by boundary conditions and singularities caused by the contact nodes still have some effect on damage identification to a certain extent. In $\mathrm{Li}$ et al.' ${ }^{25}$ work, it is mentioned that the global fluctuation of trend could be suppressed when a signal is processed by TEO. In the article, TEO is used to process damage index $D I_{m}$, and then damage index $D I_{m}^{*}$ can be obtained.

The Teager energy for a generalized discrete signal $f(n)$ can be obtained as follows

$$
T(f)=f^{2}(n)-f(n+1) f(n-1)
$$

Then the damage index $D I_{m}^{*}$ for spatial sampling points can be expressed as follows

$$
D I_{m}^{*}(n)=T\left(D I_{m}(n)\right)=D I_{m}^{\mathbf{2}}(n)-D I_{m}(n+1) D I_{m}(n-1)
$$

$D I_{m}^{*}$ results of case SD3 and case MD5 are shown in Figure 14(a) and (b). Comparing Figure 5(c) with Figure 14(a), it is observed that the fluctuations caused by boundary conditions are suppressed effectively by processing $D I_{m}$ with TEO, and the singularity caused by contact nodes is also suppressed. It demonstrates that TEO could suppress the fluctuations caused by boundary conditions and contact points effectively for the single damage identification.

However, from Figure 14(b), only damage 1 could be identified by $D I_{m}^{*}$. In the $D I_{m}^{*}$ results, the fluctuations caused by both boundary conditions and damage 2 are shielded. Because the singularity caused by damage 2 is much smaller than damage 1 , it is easy to be shielded.

The results reveal that TEO plays an important role in suppressing the effect of boundary conditions and contact points on damage identification. However, it is not suitable for multiple damages identification with different extent.

\section{Comparison with GSM-TEO method}

In $\mathrm{Li}$ et al.'s ${ }^{25}$ work, a damage index based on ULS, that is, GSM-TEO method is proposed to identify damage for composite SPTCs. To compare Li's work with the present method, the damage index DIP (damage index pattern obtained only by GSM) and IDIP (improved damage index pattern obtained only by the GSM-TEO method) proposed by $\mathrm{Li}$ is also applied on the metallic SPTC model in the work. And the results of damage cases SD3, MD1, and MD4 are shown in Figure 15(a) to (c). 

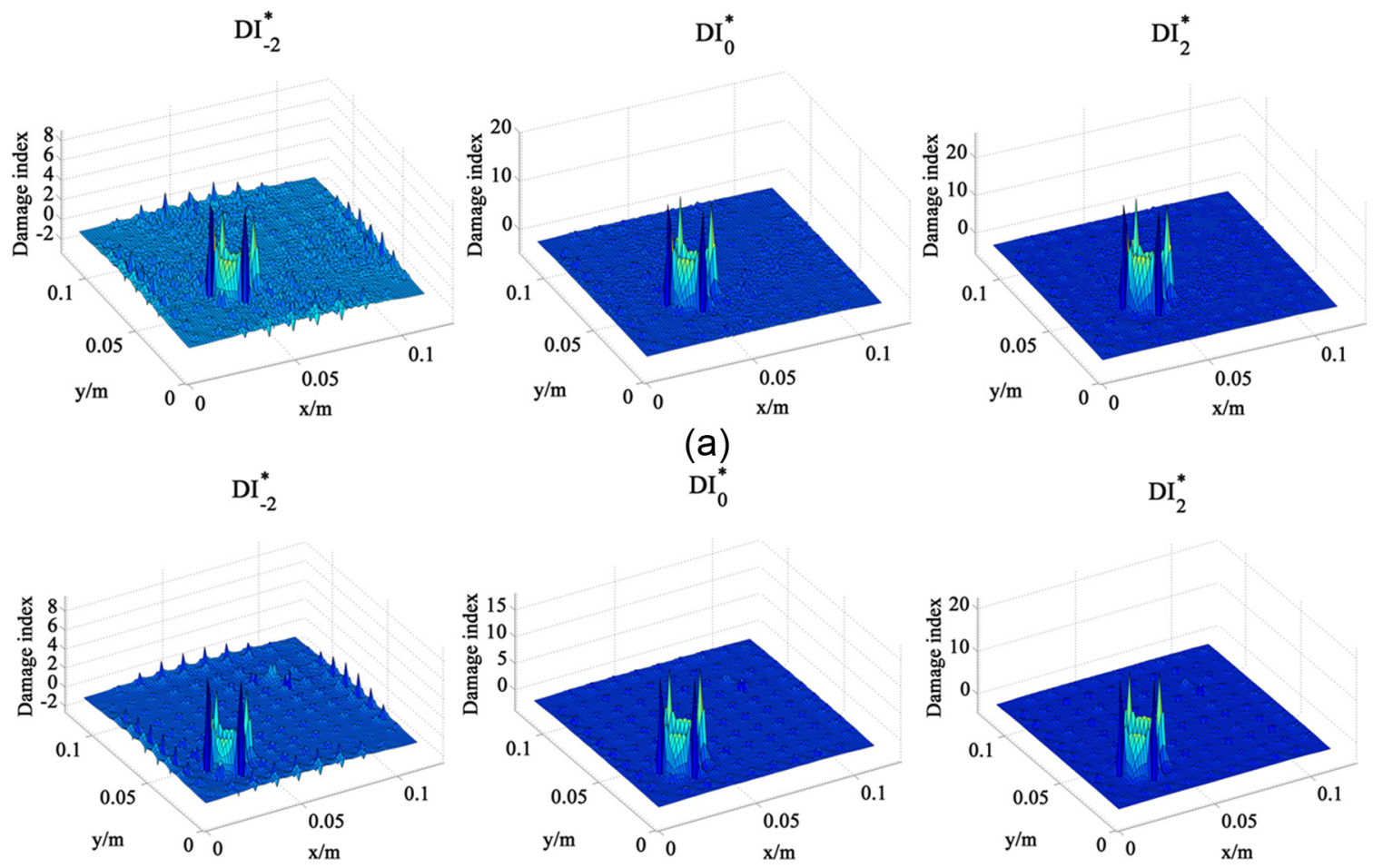

(b)

Figure 14. $D I_{m}$ results: (a) SD3 and (b) MD5.

It is worth mentioning that the four bars of a composite pyramidal truss core in Li's model do not have the common node. However, the four bars of a truss cell in the present model share the common node. Therefore, the singularity caused by contact nodes in the present model is more serious than Li's model. Normally, the common nodes property of a truss core is beneficial for the load transportation.

The damage index IDIP is very effective in identifying the damages of composite SPTCs. However, Figure 15(a) to (c) shows the damage identification results of IDIP for the present model are not as effective. The singularities caused by the contact points are so great that the damages cannot be identified. Because the singularities caused by the contact nodes in the present model are more serious than Li's model. Meanwhile, the damage index proposed by $\mathrm{Li}$ et al. includes the curvature of flexible matrix, which is more sensitive to the effect of the contact nodes. Therefore, the real damage is easily shielded by singularities caused by the contact nodes.

Comparing Figure 5(c) with Figure 15(a), it is found that the index DIP and IDIP are more effective in suppressing the fluctuations caused by boundary conditions than $D I_{m}$. This advantage could be used to improve the index $D I_{m}$ in the future work.

\section{Conclusion}

Considering the effect of damages on both high-order modes and low-order modes, a baseline-free damage identification method is presented for metallic SPTCs in the article. Numerical simulation and experimental validation are conducted to demonstrate the effectiveness of the proposed method. Based on the results, some conclusions could be obtained.

As we know, effects of different damage styles on different order mode are diverse in thousands of ways. Therefore, in damage identification of SPTCs, it is necessary to consider the effect of damages on both loworder modes and high-order modes.

The proposed damage index $D I_{m}$ has included both low-order modes and high-order modes and could identify both single damage and multiple damages effectively and it could also reflect the extent of damage in multiple damage identification. However, more effective damage extent identification method must be developed to judge the accurate extent of damage for metallic SPTCs.

Changing parameter $m$ is beneficial for detecting different damage styles. And $D I_{m}$ results in case of different $m$ should be combined together for multiple damages identification. 

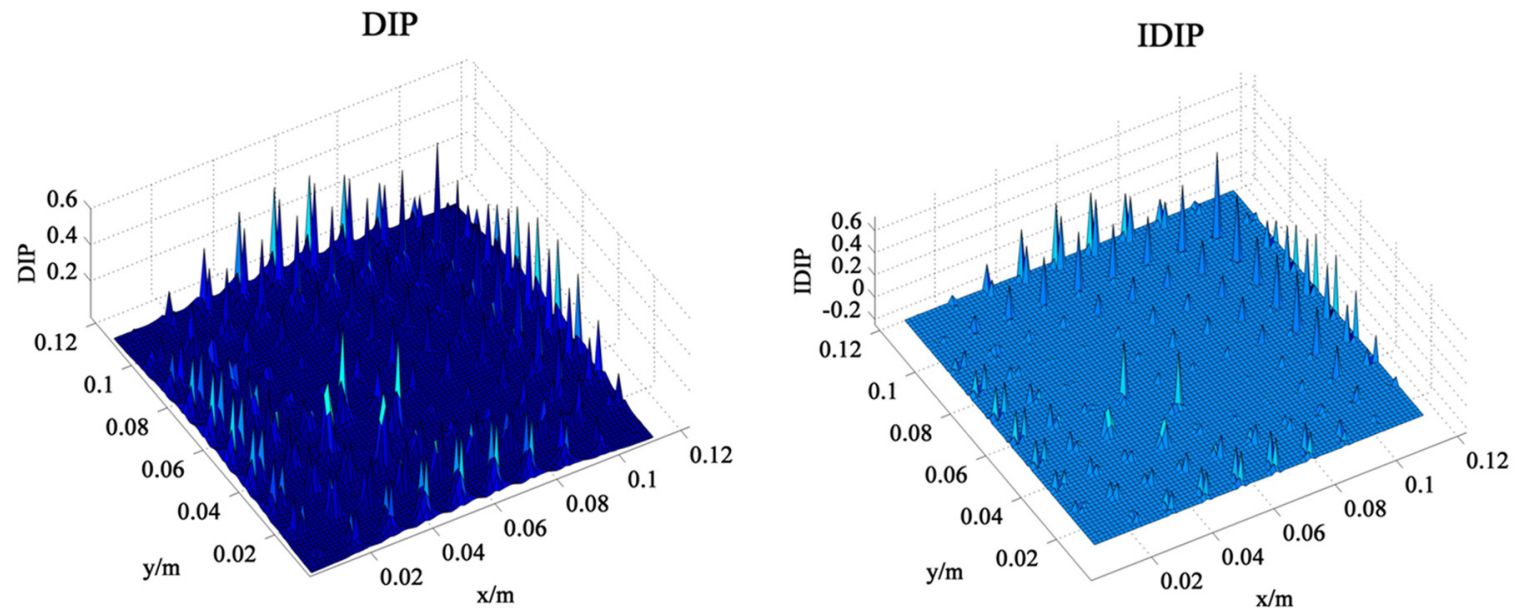

(a)
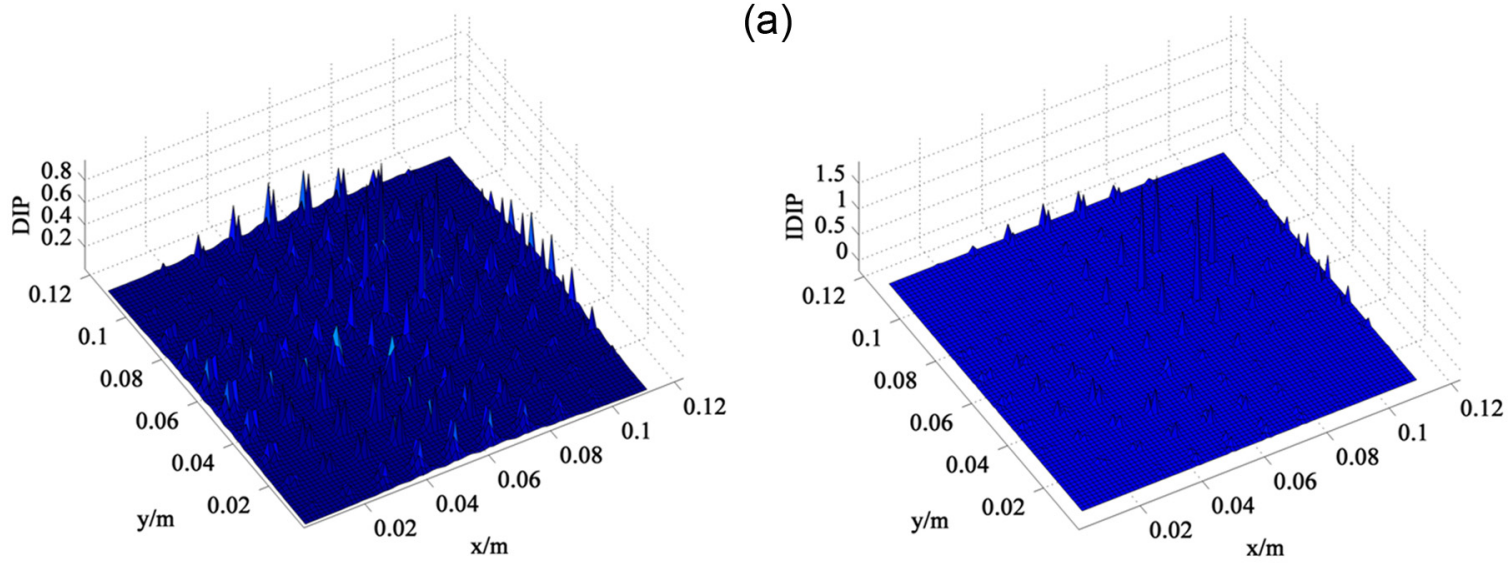

(b)

IDIP

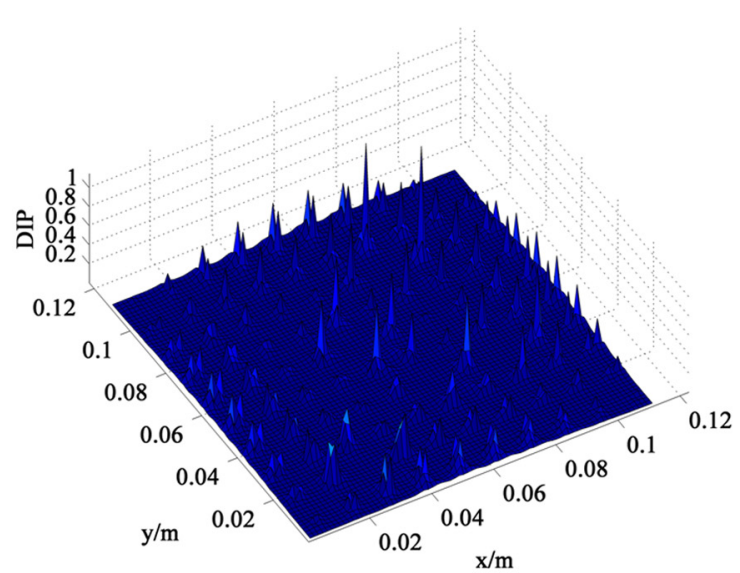

(d)

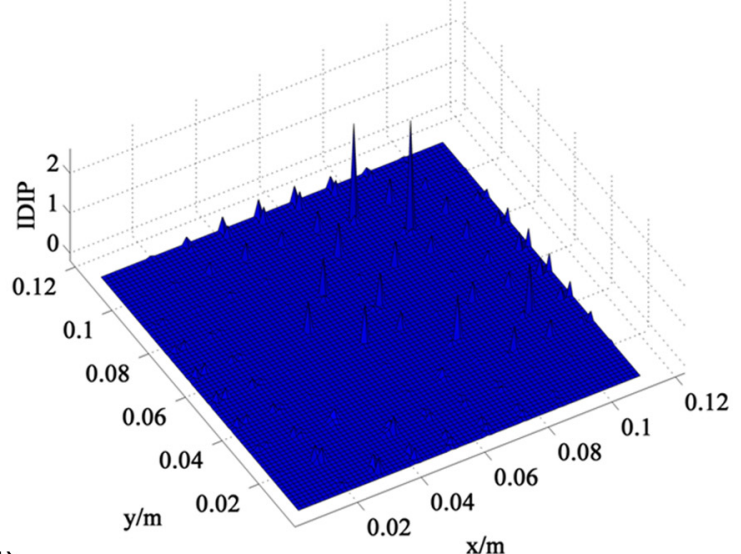

)

Figure 15. DIP and IDIP results: (a) SD3, (b) MDI, and (c) MD4.

According to $D I_{m}^{*}$ results, it is found that TEO could effectively suppress the fluctuations caused by both boundary conditions and contact joints. However, TEO is not suitable for multiple damages with different extent.

\section{Declaration of Conflicting Interests}

The author(s) declared no potential conflicts of interest with respect to the research, authorship, and/or publication of this article. 


\section{Funding}

The author(s) disclosed receipt of the following financial support for the research, authorship, and/or publication of this article: This research is supported by the National Natural Science Foundation of China (Grant Nos 11472276, 11332011, and 11502268) and Defense Industrial Technology Development Program.

\section{References}

1. Quek ST, Tua PS and Wang Q. Detecting anomalies in beams and plate based on the Hibert-Huang transform of real signals. Smart Mater Struct 2003; 12(3): 447-460.

2. Xu QS. Impact detection and location for a plate structure using least squares support vector machines. Struct Health Monit 2014; 13(1): 5-18.

3. $\mathrm{Hg} \mathrm{A.} \mathrm{Analysis} \mathrm{and} \mathrm{design} \mathrm{of} \mathrm{structural} \mathrm{sandwich} \mathrm{panels.}$ Oxford: Pergamon Press, 1969.

4. Chiras S, Mumm DR, Evans AG, et al. The structural performance of near-optimized truss core panels. Int $J$ Solids Struct 2002; 39: 4093-4115.

5. Wadley HNG, Fleck NA and Evans AG. Fabrication and structural performance of periodic cellular metal sandwich structures. Compos Sci Technol 2003; 63: 2331-2343.

6. Evans AG, Hutchinson JW, Fleck NA, et al. The topological design of multifunctional cellular metals. Prog Mater Sci 2001; 46: 309-327.

7. Wang J, Lu TJ, Woodhouse J, et al. Sound transmission through lightweight double-leaf partitions: theoretical modeling. J Sound Vib 2005; 286(4-5): 817-847.

8. Zumpano G and Meo M. Damage detection in an aircraft foam sandwich panel using nonlinear elastic wave spectroscopy. Comput Struct 2008; 86: 483-490.

9. Panopoulou A, Loutas T, Roulias D, et al. Dynamic fiber Bragg gratings based health monitoring system of composite aerospace structures. Acta Astronaut 2011; 69: 445-457.

10. Yuan W, Wang X, Song HW, et al. A theoretical analysis on the thermal buckling behavior of fully-clamped sandwich panels with truss cores. J Therm Stresses 2014; 37: 1433-1448.

11. Yuan W, Song HW, Wang X, et al. Experimental investigation on thermal buckling behavior of fully-clamped truss-core sandwich panels. AIAA J 2015; 53(4): 948-957.

12. Russell B and Hilary BS. Imperfection sensitivity of pyramidal core sandwich structures. Int J Solids Struct 2007; 44: 4690-4706.

13. Evans AG, Hutchinson JW and Ashby MF. Multifunctionality of cellular metal systems. Prog Mater Sci 1998; 43(3): 171-221.
14. Wallach JC and Gibson LJ. Defect sensitivity of a 3D truss material. Scripta Mater 2001; 45: 639-644.

15. Hu JS and Hwu C. Free vibration of delaminated composite sandwich beams. AIAA J 1995; 33(10): 1911-1918.

16. Kim HY and Hwang W. Effect of debonding on natural frequencies and frequency response functions of honeycomb sandwich beams. Compos Struct 2002; 55: 51-62.

17. Sokolinsky VS, Bremen HF, Lesko JJ, et al. Higher-order free vibrations of sandwich beams with a locally damaged core. Int J Solids Struct 2004; 41: 6529-6547.

18. Burlayenko VN and Sadowski T. Influence of skin/core debonding on free vibration behavior of foam and honeycomb cored sandwich plates. Int J Nonlinear Mech 2010; 45(10): 959-968.

19. Buket OB and Srinivasa T. An experimental investigation of free vibration response of curved sandwich beam with face/core debond. J Reinf Plast Comp 2010; 29: 3208-3218.

20. Lou J, Wu LZ, Ma L, et al. Effects of local damage on vibration characteristics of composite pyramidal truss core sandwich structure. Compos Part B: Eng 2014; 62: 73-87.

21. Hu HW, Wang BT, Lee CH, et al. Damage detection of surface cracks in composite laminates using modal analysis and strain energy method. Compos Struct 2006; 74 : 399-405.

22. Kumar M, Shenoi RA and Cox SJ. Experimental validation of modal strain energies based damage identification method for a composite sandwich beam. Compos Sci Technol 2009; 69: 1635-1643.

23. Tian SX, Chen ZM, Chen LL, et al. Numerical analyses on influence of damage configuration on vibration parameters for lattice sandwich plate. Int $J$ Appl Electrom 2010; 33: 1565-1572.

24. Zhu KG, Chen MJ, Lu QH, et al. Debonding detection of honeycomb sandwich structures using frequency response functions. J Sound Vib 2014; 333: 5299-5311.

25. Li B, Li Z, Zhou J, et al. Damage localization in composite lattice truss core sandwich structures based on vibration characteristics. Compos Struct 2015; 126: 34-51.

26. Andrzej K. Vibration-based spatial damage identification in honeycomb-core sandwich composite structures using wavelet analysis. Compos Struct 2014; 118: 385-391.

27. Ratcliffe CP. Damage detection using a modified Laplacian operator on mode shape data. J Sound Vib 1997; 204: 505-517.

28. Yoon MK, Heider D, Gillespie JW, et al. Local damage detection using the two-dimensional gapped smoothing method. J Sound Vib 2005; 279: 119-139. 